
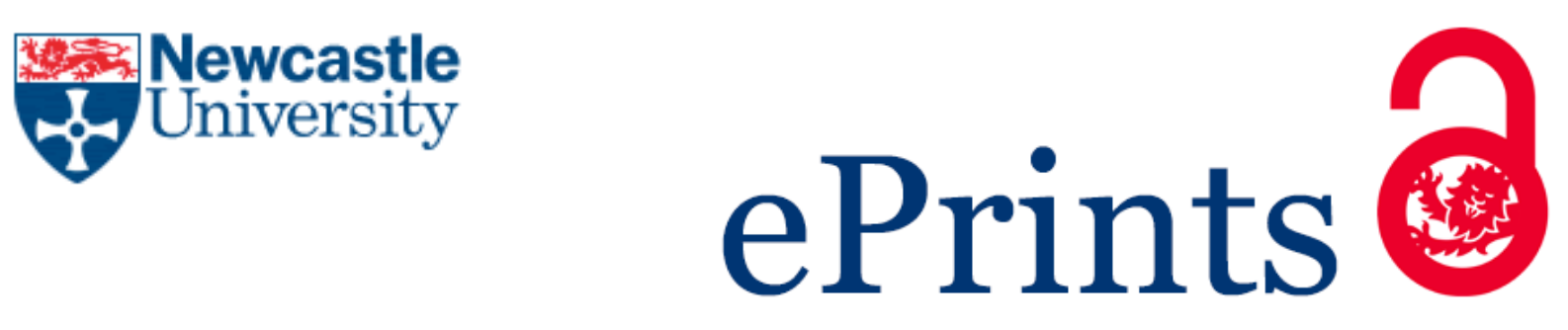

Papastavrou VT, Borooah S, O'Brien JM, Ray-Chaudhuri N, Dhillon B, Vieira R, Browning A.

Cataract surgery in patients with late-onset retinal degeneration. Journal of Cataract and Refractive Surgery 2017, 43(8), 1036-1043.

\title{
Copyright:
}

(C) 2017. This manuscript version is made available under the CC-BY-NC-ND 4.0 license

DOI link to article:

https://doi.org/10.1016/j.jcrs.2017.05.041

Date deposited:

$02 / 10 / 2017$

Embargo release date:

01 August 2018

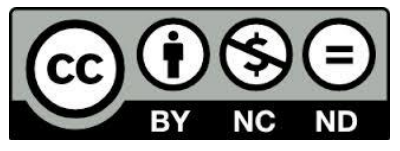

This work is licensed under a

Creative Commons Attribution-NonCommercial-NoDerivatives 4.0 International licence 


\section{Cataract surgery in patients with Late-Onset Retinal Degeneration (L-ORD)}

Vasileios T. Papastavrou MD, MRCOphth, FEBO,1

Shyamanga Borooah FRCOphth, PhD 3,

Jill M O’Brien BA (Hons)1

Neeta Ray- Chaudhuri FRCOphth, FRCS (Ed), FRCS (Glas)1

Baljean Dhillon. FRCOphth 3

Rute V Vieira 4

Andrew C Browning, BM, PhD. 1,2

1) Newcastle Eye Centre, Royal Victoria Infirmary, Newcastle upon Tyne, NE1 4LP, UK

2) Institute of Genetic Medicine, International Centre for Life, Newcastle University, Newcastle upon Tyne, NE1 3BZ, UK.

3) Department of Ophthalmology, Princess Alexandra Eye Pavilion, Chalmers Street, Edinburgh, EH3 9HA, UK

4) Institute of Health and Society, Faculty of Medical Sciences, Newcastle University, Newcastle upon Tyne, NE2 4AX, UK

Corresponding author: Andrew C Browning, Newcastle Eye Centre, Royal Victoria Infirmary, Newcastle upon Tyne, NE1 4LP

Tel no +44 1912820474

Fax No +44 1912826269

e-mail: andrew.browning@nuth.nhs.ukDr Kathryn White and Tracey Davey of Newcastle University Electron Microscopy Research Services performed the scanning electron microscopy on the lens caspsule samples.

Conflict of interest statement: : The authors state that there are no actual or potential conflicts of interest in relation to this manuscript 
Abstract

Purpose:

To review the surgical and visual outcomes in a series of patients with long anterior lens zonules (LAZs) associated with late onset retinal degeneration (L-ORD), who underwent phacoemulsification cataract surgery.

Setting:

Newcastle Eye Centre, Royal Victoria Infirmary, Newcastle upon Tyne., UK

Design:

Retrospective case series.

Methods:

Inclusion criteria were patients with genetically confirmed L-ORD requiring cataract surgery. Perioperative data relating to surgery was collected. Additionally, bestcorrected visual acuity and retinal imaging data was recorded. Selected lens capsules were examined by immunohistochemistry or scanning electron microscopy (SEM).

Results:

Eleven eyes of seven patients were included. The LAZs made capsulorhexis challenging, however it was completed safely in all cases. There were no intra or post-operative issues with lens stability. Visual acuity improved post operatively in those cases with intact foveal photoreceptors and RPE. Over the longer term, visual 
acuity slowly declined due to progressive atrophy of the macular photoreceptors and RPE. The majority of patients noticed a subjective improvement in vision, even those with advanced disease at baseline. Immunohistochemistry showed that the C1QTNF5 protein was expressed within the lens capsule epithelial cells, while SEM of the LAZs showed them to be smaller in diameter to normal anterior lens zonules and to be composed of a helix of fibres.

\section{Conclusion}

In this small series of L-ORD subjects, cataract surgery was successfully performed without long term complications involving intraocular lens stability. Objective improvement in visual acuity appears to be limited to those patients with good foveal photoreceptor architecture.

\section{What was known?}

Late onset retinal degeneration (L-ORD) is associated with long anterior lens zonules (LAZs), which reduce the zonule free area of the anterior lens capsule available to perform capsulorhexis during cataract surgery.

Long anterior lens zonules may increase the rate of complications during cataract surgery.

\section{What this paper adds}

Capsulorhexis can be safely performed in patients with LAZs associated with L-ORD The lens remains stable during phacoemulsification cataract surgery and long term follow up demonstrates that the intraocular lens remains well centred. 
Improvement in visual acuity appears to be limited to those patients with good foveal photoreceptor and RPE architecture, requiring careful pre-operative retinal assessment.

\section{Introduction}

Late-onset retinal degeneration (L-ORD) is a rare progressive autosomal dominant rod/cone dystrophy (OMIM 605670) caused by a mutation in the gene C1QTNF5, leading to a substitution (Ser163Arg) in the protein ${ }^{1,2}$. The normal role of C1QTNF5 is still unclear, however it is highly expressed within the eye in the retinal pigment epithelium (RPE), ciliary body and lens epithelial cells ${ }^{2,3}$. Disease onset is usually in the fourth decade, with the patients reporting nyctalopia, but with little in the way of retinal findings except some fine macular drusen. The disease progresses to first macular and then peripheral chorio-retinal atrophy with most patients registered blind by the seventh decade ${ }^{4}, 5$. A consistent finding in patients with L-ORD are long anterior lens zonules (LAZs) ${ }^{2}$. It is not known if these LAZs are structurally important or are accessary zonules. The LAZs reduce the zonule free area available for capsulorhexis during cataract surgery and may therefore make surgery technically challenging ${ }^{6,7}$. Because the symptoms of L-ORD present later in life, removal of their age-related cataracts may be contemplated in an attempt to improve their declining visual function. In retinitis pigmentosa (RP), another type of rod/cone dystrophy, cataract surgery for predominantly posterior sub-capsular opacities may be complicated by zonular weakness causing intraoperative zonule dialysis, late phacodonesis and lens subluxation ${ }^{8-10}$. Visual outcome after cataract surgery in patients with RP is also affected by the state of macular photoreceptors in particular the status of the foveal ellipsoid zone ${ }^{11,12}$. We describe a retrospective series of 11 eyes of 7 patients with L-ORD who underwent phacoemulsification cataract surgery, with particular emphasis on the peri-operative findings, the potential visual benefits of the procedure and the long term stability of the intra-ocular lens. 


\section{Material and methods:}

In this retrospective case series, the clinical records of patients with L-ORD who had previously undergone cataract surgery were reviewed. All patients had been diagnosed with L-ORD by the carriage of the Ser163Arg mutation in C1QTNF5. All aspects of the study were approved by the local research ethics committee and all procedures used conformed to the tenets of the Declaration of Helsinki.

The best corrected visual acuity (BCVA) was measured prior to, at one month postsurgery, at 12 months post- surgery and at the most recent clinic visit. At the 1 month post-operative visit, the patient was also asked whether they had noticed any subjective improvement in the quality of their corrected vision. Where the BCVA was measured as Snellen acuity, it was converted to LogMAR. The type of cataract was noted. The patient's disease was graded using the system of Borooah et $\mathrm{al}^{4}$. In addition, the status of the macula ellipsoid zone as determined by spectral domain optical coherence tomography (OCT) was noted prior to surgery using the system of Nakamura et $\mathrm{al}^{12}$. Briefly, this is a grading system that has been used as a surrogate measure of foveal and parafoveal photoreceptor health in patients with retinitis pigmentosa, another type of rod cone dystrophy. In grade 1, the sub/para foveal ellipsoid zone is not visible, in grade 2 , the ellipsoid zone is abnormal/discontinuous and in grade 3 , the line is normal. At the most recent clinic visit, any evidence of lens subluxation was noted. As L-ORD is a rare retinal dystrophy and the number of eyes included in the study is limited, the main analysis of the overall results was qualitative with no hypothesis testing. BCVA between surgery related time points and the grade of the ellipsoid zone defects were described and compared graphically and numerically using appropriate measures of location and spread (median, interquartile range and range).

The zonule free zone was measured in a number of different patients with L-ORD, taking part in a separate observational study. Retroillumination anterior segment photographs (Haag-Streit BX900 slit lamp camera, Harlow, Essex, UK) were taken that allowed simultaneous visualisation of the LAZs and the tips of measuring 
calipers, set to $4 \mathrm{~mm}$, which were held at the limbus. This ensured that the calipers were in the plane of the anterior lens. Fig 1 is an anterior segment photograph of a typical patient with L-ORD, demonstrating the reduced zonule free area and iris margin atrophy caused by long anterior lens zonules.

Cataract surgery was performed under sub-Tenon's local anaesthetic by three of the authors (VTP, NRC and ACB) who carried out standard phacoemulsification and implantation in the bag of an intraocular lens (AcrySof SN60WF [Alcon, Camberley, UK] or Hoya iSert 250 [Spectrum, Macclesfield, UK]) through a superior or temporal corneal incision.

After filling the anterior chamber with viscoeleastic (Bio-Hyalur, SD Healthcare, Irlam, UK), the zonule free area was noted and the capsulorhexis was started centrally with a vertical puncture of the capsule using either a pre-formed $25 \mathrm{G}$ cystotome (Altomed,Ltd, Bolden, Tyne and Wear, UK), or a bent 29G U-100 insulin needle (Beckton Dickinson, Oxford, UK). An approximately $4 \mathrm{~mm}$ radial incision is made from this puncture site extending centrifugally, into and parallel with the anterior zonular fibres. A small triangular-shaped flap was lifted using the tip of the needle and carefully turned over, overcoming the resistance of the attached zonules. The flap was then grasped with capsulorhexis forceps and a gradual spiral movement used to complete an approximately $5-5.5 \mathrm{~mm}$ diameter capsulorhexis ${ }^{13}$. Particular care was taken by frequently re-grasping the edge of the free flap to reduce the risk of the capsulorhexis running out. Enhanced visualisation of the capsule and LAZs was undertaken using BioBlue PFS (SD Healthcare, Irlam, UK) if it was felt necessary by the surgeon. After hydrodissection, phacoemulsification surgery was competed using an Oertli OS3 phaco machine (Oertli instruments AG,,Berneck, Switzerland ) and a single piece posterior chamber intraocular lens inserted and standard post-operative care was initiated. Any complications or evidence of intraoperative phacodonesis were noted. Selected anterior capsules with attached zonules were immediately placed in either buffered formalin solution (10\%) for immunohistochemistry or $2 \%$ gluteraldhyde in Sorenson's phosphate buffer for scanning electron microscopy. 
Immunohistochemistry

Samples were embedded in paraffin wax after dehydration in a graded ethanol series. Sections of the capsule were cut at 3 micron thickness, mounted on Superfrost Plus glass slides (Menzel-Glaser, Braunschweig, Germany) deparaffinised in xylene and exposed to a rabbit anti-C1QTNF5 (LS-B1343, LSBio, Seattle, USA) at a 1:250 dilution for 2 hours at room temperature. Following multiple washes with Bond wash buffer solution (Leica Biosystems, Newcastle upon Tyne, UK), antibody binding was visualised using 3,3'diaminobenzidine (DAB) as a substrate for peroxidase, resulting in a positive brown colour. A negative control, where the antibody was omitted and replaced with normal goat serum was also examined. Sections were counter-stained with haematoxylin to aid tissue visualisation

Scanning electron microscopy

After the samples had been fixed overnight in $2 \%$ glutaraldehyde in Sorenson's phosphate buffer, they were rinsed in several changes of buffer, then dehydrated through a graded series of ethanol. Once in $100 \%$ ethanol, final dehydration was carried out by critical-point drying with carbon dioxide. Samples were mounted on an aluminium stub, coated with $15 \mathrm{~nm}$ gold and examined with a Cambridge Stereoscan 240 SEM. 


\section{Results}

Eleven eyes of 7 patients with L-ORD underwent uncomplicated phacoemulsification cataract surgery. The median age of the patients was 69 years (range 65-79) and 10 out of 11 cataracts were of the nuclear sclerotic type. The median ocular axial length was $23.0 \mathrm{~mm}$ (range 21.85-24.20 mm). The median pre-operative spherical equivalent was 0.5 dioptres (range -3.5 to +3.0). At the time of surgery, 8 out of 11 patients had stage 3 disease and 3 out of 11 had stage 2 disease. The surgery was performed by 3 different experienced surgeons and Bio Blue was used to stain the capsule in 2 eyes to better visualise the zonules during capsulorhexis. During the capsulorhexis step, all surgeons felt that there was a difficulty in turning the capsular flap over at the start of the procedure and a marked resistance of the capsule to tear, due to the strength of the zonules that needed to be broken to allow the capsulorhexis to proceed. There was also a distinct tendency of the curvilinear process to veer towards the equator and special care was required to avoid this. The desired size of capsulorhexis (approximately $5.5 \mathrm{~mm}$ ) was achieved in all patients, allowing safe surgery to proceed and to provide a small overlap of the capsulorhexis and the edge of the lens optic. In all cases, the rest of the surgery was uncomplicated with no evidence of intra-operative lens instability, anterior chamber deepening or zonular dialysis. No capsular tension rings were inserted. Table 1 summarises the patient demographics, the ellipsoid zone grade and the BCVA measured pre-op, at 4 weeks, at 12 months post op and at the most recent clinic visit. In a separate group of patients with L-ORD not undergoing surgery, the mean central zonule free area of 15 eyes of 11 patients was measured as $3.0 \mathrm{~mm}$ (range 2.3-5.2mm). The mean age of these patients was 59 years (range 36 to 71 years). Interestingly, in some patients, the LAZs were seen to be isolated, with no connection to more peripheral anterior lens zonules.

Three out of 11 eyes demonstrated an objective improvement in BCVA at 1 month post op (two eyes had grade 3 [intact] ellipsoid zones and one was grade 2 [interrupted] ellipsoid zone) The BCVA of 4 eyes was unchanged at 1 month post-op (one grade 3 [intact] ellipsoid zone and three grade 1 [absent] ellipsoid zones). Three 
eyes demonstrated a deterioration in BCVA. Two of these eyes had pre-existing poor vision (absent ellipsoid zones). No recognised cause of post- operative visual acuity deterioration such as cystoid macular oedema could be found to account for the worsening of vision. The third patient (grade 3 or intact ellipsoid zone) developed a macular involving choroidal neovascular membrane. This eye was subsequently treated with intravitreal Aflibercept, but responded poorly. Although only 3 out of 11 eyes demonstrated an objective improvement in vision at 1 month post op, 7 out of 11 eyes were recorded as having a subjective improvement. Unfortunately, by 12 months, 5 out of 11 eyes demonstrated a reduction in vision compared with the 1 month level, due to progression of RPE and photoreceptor atrophy at the fovea, when assessed by clinical examination and by macular OCT and autofluorescence imaging (data not shown). The visual acuity of 5 eyes were stable at 12 months, although some of these already had poor visual acuity at baseline due to foveal RPE and photoreceptor atrophy. At the last follow up visit (mean 40 months, range: 6 to 84 months) the visual acuity had deteriorated further in most eyes, again due to progressive macular RPE and photoreceptor atrophy. The overall distribution of visual acuity at each time point is represented in Fig 2. Median visual acuity improved at 1 month post op ( $0.6 \log M A R)$ when compared with baseline $(0.8$ logMAR), however, it deteriorated considerably at 12 months post op (1.2 logMAR). While the range is similar at baseline and 1 month post op, BCVA is consistantly worse at 12 months. Fig 3 shows the range and the median visual acuity over time, according to grade of the ellipsoid zone defects. As expected, pre-op visual acuity differs between grades, but there is a tendency of worsening vision at 12 months for grades. 2 and 3 (interrupted and intact ellipsoid zones respectively) At the last follow up visit, all intra ocular lenses remained centred and no eyes had required a YAG capsulotomy. Fig 4a and b demonstrates the representative Goldmann visual fields of patients with mild (early stage 2 ) and severe (advanced stage 3 ) L-ORD respectively 
Immunohistochemistry

Two anterior lens capsules from patients with molecularly confirmed L-ORD and 1 normal control were stained for C1QTNF5 protein. This demonstrated expression of C1QTNF5 within the cytoplasm of the capsule epithelial cells (fig 5a). No staining for C1QTNF5 was demonstrated in the negative control (fig 5b). No attached zonules were seen in the sections examined.

SEM

Two anterior lens capsules from patients with molecularly confirmed L-ORD were examined by scanning electron microscopy. Macroscopic examination of the capsule showed that while some zonules remained attached, many had been lost either during surgery or processing. Detailed examination showed that before insertion, the free zonules were between 2 and 5 microns in diameter and were composed of bundles of fibres, wound together in a spiral (figs 6a and 6b) Near the point of insertion, the zonules taper down to approximately1 to 2 microns in diameter (fig 7) and Individual fibrils can be seen attaching the underside of the zonule, to the capsule surface (fig 8). The zonule is then seen to blend with the surface of the capsule (fig 9). 


\section{Discussion:}

In humans, the developing lens zonules are recognisable at 10 weeks of gestation, where they are termed the tertiary vitreous ${ }^{14}$. They are thought to originate from the non-pigmented ciliary epithelium and their main roles are to hold the lens in place and to mediate the forces of accommodation. In the normal state, the anterior lens zonules insert into the lens capsule approximately $1.5 \mathrm{~mm}$ anterior to the equator of the lens ${ }^{15}$. In L-ORD, the anterior lens zonules insert further forward on the capsular surface, reducing the size of the capsular zonule free area (mean $2.7 \mathrm{~mm}$ in this series). This finding has also been described occasionally in elderly, hyperopic females, where it is associated with a short axial length, pigment dispersion and possibly plateau iris configuration ${ }^{16-18}$. In a study of these patients, $50 \%$ of cases had a zonule free area of less than $3 \mathrm{~mm}^{19}$. The protein, C1QTNF5 is known to be highly expressed in the human ciliary body as well as the $\mathrm{RPE}^{2}$ and in mice it is expressed at the junction of the pigmented and non- pigmented ciliary epithelium ${ }^{3}$. It is therefore feasible that in patients with L-ORD, who carry the disease causing mutation, zonule development or function may be abnormal. These LAZs could pose a number of potential problems during and after cataract surgery such as; difficulty in achieving a satisfactory size of capsulorhexis, lens instability during surgery due to widespread disruption of the insertion of the LAZs and post-operative lens subluxation $6,7,20$

This study reviewed the surgical and visual outcomes of 11 eyes of 7 patients with LORD who underwent phacoemulsification cataract surgery between 2008 and 2016 in a single unit. In all cases, a satisfactory capsulorhexis of between 5 to $5.5 \mathrm{~mm}$ was achieved although difficulty was noted in turning over the initial flap and there was a marked resistance to tearing and a tendency of the capsulorhexis to veer "out" which required the use of numerous small controlled tearing movements rather than the more usual sweeping motion. Some authors have recommended staining the capsule and zonules with either trypan blue or ICG to enhance visualisation of idiopathic LAZs in order to prevent complications ${ }^{6,7}$, however we didn't find any advantage to using this technique in the 2 cases that we used it on. After the capsulorhexis stage, the rest of the surgery was routine. There was no evidence of anterior chamber deepening or lens instability. This would suggest that although the capsular attachments of the LAZs are broken during capsulorhexis, there is sufficient 
support provided by the equatorial and posterior zonules to prevent subluxation. In addition, in some patients, the LAZs were seen to be isolated, with no connection to more peripheral anterior lens zonules, a similar finding to those described by Roberts and Wilensky in patients with idiopathic LAZs ${ }^{21}$. During long term follow up (7 years in one case), the lenses of all patients remained well centred. Interestingly, Roberts et al recently published a retrospective case series on the surgical outcomes of 29 eyes of 19 patients from an African American sub- population with LAZs. There was no mention of any type of retinal dystrophy in their patients. Although the underlying cause of LAZs in their cohort is probably different to our LORD patients, the authors did not report any surgical complications or any episodes of lens decentration (up to 6.8 years of follow up) ${ }^{22}$. This study supports our surgical findings and suggests that the LAZs are not critical for lens stability.

Scanning electron microscopy (SEM) of normal anterior lens zonules show them to initially consist of bundles of fibres approx. 25-60 microns in diameter ${ }^{23}$. Just prior to where they insert, they rapidly spread out on the capsule surface as 5-10 micron bundles, which continue to decrease in size ${ }^{16}$. They end as 0.3-0.4 micron fanning fibres whose terminal fibrils blend into the surface of the capsule. ${ }^{15,23}$ When the capsules along with attached zonules from patients with L-ORD were examined by SEM, the zonules were found to be narrower in diameter when compared to the published diameter of normal anterior zonules. They were also found to be composed of fibrils wound in a helical fashion which has not previously been reported in normal anterior zonules. To date, only the posterior segment of a globe from a patient with L-ORD has been examined, which demonstrated the wellrecognised retinal and RPE atrophy ${ }^{24}$. It would be interesting to study the anterior segment of a donor globe to determine more comprehensively whether the ciliary body and the equatorial/posterior zonules are normal and to examine the LAZs more fully.

In the short term, there was an objective improvement in the BCVA of 3 out of 11 eyes. All 3 eyes had either an intact or discontinuous sub foveal ellipsoid zone, implying reasonably well preserved photoreceptor health. A similar finding has been described in patients with retinitis pigmentosa undergoing cataract surgery, where a 
better visual outcome has been found in those patients with an intact sub foveal ellipsoid zone ${ }^{12}$. Disappointingly, in the other 8 eyes, no objective improvement in short term vision was noted and one eye even developed of sub foveal choroidal neovascular membrane within 4 weeks of surgery, that responded poorly to treatment. The majority of these cases had more advanced disease with macular atrophy and an absent ellipsoid zone. Interestingly, at 4 weeks post op, the majority of eyes demonstrated a subjective improvement in the quality of vision, even some eyes with absent foveal architecture. This suggests that cataract surgery may be beneficial in patients with L-ORD, at least in the short term, even in those with more advanced disease. In the longer term, of those patients with reasonable vision at their first post op visit (logMAR 0.3-0.6) only 1 patient out of 6 maintained stable vison at 12 months post op. The remaining five patients lost on average 0.4 logMAR units due to progression of their macular atrophy. No cases of posterior capsule opacification occurred that required intervention. Macular atrophy continued to progress to the extent that at their last follow up visit (mean follow up duration 44 months), the visual acuity had fallen to 1.4 logMAR in 9 out of 11 eyes. In the two eyes that had maintained their vison, follow up was relatively short (27 and 6 months respectively) compared with the other cases

In conclusion, cataract surgery in patients with LAZs secondary to L-ORD appears to be a safe procedure with maintenance of long-term lens stability. Objective improvement in visual acuity appears to be limited to patients with milder disease who maintain good foveal photoreceptor architecture. In patients with more advanced disease who have marked macular involvement, objective improvement in vison is limited, however some patients did report subjective improvement. 
References

1. Hayward C, Shu X, Cideciyan AV, et al. Mutation in a short-chain collagen gene CTRP5, results in extracellular deposit formation in late-onset retinal degeneration: a genetic model for age-related macular degeneration. Hum Mol Genet 2003;12:2657-2667.

2. Ayyagari R, Mandal MN, Karoukis AJ, et al. Late-onset macular degeneration and long anterior lens zonules result from a CTRP5 gene mutation. Invest Ophthalmol Vis Sci 2005;46:3363-3371.

3. Mandal MN, Vasireddy V, Reddy GB, et al. CTRP5 is a membraneassociated and secretory protein in the RPE and ciliary body and the S163R mutation of CTRP5 impairs its secretion. Invest Ophthalmol Vis Sci 2006;47:5505-5513.

4. Borooah S, Collins C, Wright A, Dhillon B. Late-onset retinal macular degeneration: clinical insights into an inherited retinal degeneration. $\mathrm{Br} \mathrm{J}$ Ophthalmol 2009;93:284-289.

5. Cukras C, Flamendorf J, Wong WT, et al. Longitudinal structural changes in late-onset retinal degeneration. Retina 2016;0:1-9.

6. Chen S, Glover N, Patel CK. Trypan blue staining of anteriorly placed zonules in patients with pigment dispersion. J Cataract Refract Surg 2004;30:2462-2463.

7. Kelty PJ, Snyder ME, Schneider S. Indocyanine green staining of anteriorly placed zonules. J Cataract Refract Surg 2003;29:2229-2231. 
8. Dikopf MS, Chow CC, Mieler WR, Tu EY. Cataract extraction otcomes and he prevalence of zonular insufficiency in retinitis pigmentosa. Am J Ophthamol 2013;156:82-88.

9. Hayashi K, Hayashi H, Matsuo K, Nakao F, Hayashi F. Anterior capsule contraction and intraocular lens dislocation after implant surgery in eyes wih retinitis pigmentosa. Ophthalmology 1998;105:1239-1243.

10. Jackson H, Garway-Heath D, Rosen P, Bird AC, Tuft SJ. Outcome of cataract surgery in patients with retinitis pigmentosa. $\mathrm{Br} \mathrm{J}$ Ophthalmol 2001;85:936-938.

11. Yoshida N, Ikeda $Y$, Murakami $Y$, et al. Factors affecting visual acuity after cataract surgery in patients with retinitis pigmentosa. Ophthalmology 2015;122:903-908.

12. Nakamura $Y$, Mitamura $Y$ Hagiwara A, et al. Relationship between retinal microstructures and visual acuity after cataract surgery in patients with retinitis pigmentosa. $\mathrm{Br} \mathrm{J}$ Ophthalmology 2013;99:508-511.

13. Hasanee K, Butler M, Ahmed II. Capsular tension rings and related devices: current concepts. Curr Opin Ophthalmol 2006;17:31-41.

14. Marshall J, Beaconsfield M, Rothery S. The anatomy and development of the human lens and zonules. Trans Ophthal Soc UK 1982;102:423-440.

15. Streeton BW. Anatomy of zonular apparatus. In Duane's Fondations of clinical Ophthalmology on CD-ROM. Tasman WS, Jaegar EA eds; Philadelphia; Lippincott Williams and Wilkins, 2006; volume 1: Chapter 14.

16. Moroi SE, Sieving PA, Nouri-Mahdavi K, Schlotzer-Schrehardt U, Katz GJ. Long anterior zonules and pigment dispersion. Am J Ophthalmol 2003;136:1176-1178. 
17. Roberts DK, Ayyagari R, McCarthy B, et al. Investigating ocular dimensions in African-Americans with long anterior zonules. J Glaucoma 2013;22:393397.

18. Roberts DK, Ayyagari R, Moroi SE. possible association between long anterior lens zonules and plateau iris configuration. J Glaucoma 2008;17:393-396.

19. Roberts DK, Yang Y, Morettin CE, et al. Quantification of long anterior lens zonules and their resulting zonule-free zone sizes. Clin Exp Ophthalmol 2015; 43:773-775.

20. Lim SJ, Kang SJ, Kim HB, Kurata Y, Sakabe I, Apple DJ. Analysis of zonular-free zone and lens size in relation to axial length of eye with age. $J$ Cataract Refract Surg 1998;24:390-396.

21. Roberts DK and Wilensky J. Long anterior lens zonules. Clin Exp Ophthalmol 2012;40:764-766.

22. Roberts DK, Yang Y, Wilensky JT. Cataract surgery in African Americans with long anterior lens zonules. Clin Exp Ophthalmol 2014;42:898-900.

23. Streeten BW. The zonular insertion: a scanning electron microscopic study. Invest Ophthalmol Vis Sci 1977;16:364-375.

24. Milam AH, Curcio CA, Cideciyan AV, et al. Dominant late-onset retinal degeneration with regional variation of sub-retinal pigment epithelium deposits, retinal function and photoreceptor degeneration. Ophthalmology 2000;107:2256-2266. 
Figures

Fig 1. Peripupillary iris atrophy and long anterior lens zonules in a patient with LORD. Note the small central capsular zonule free area.

Fig 2. Graphical representation of the distribution of BCVA at pre-op, 1 month post op and 12 months post op, showing the range, interquartile range and median BCVA.

Fig 3. Graphical representation of the median and range of BCVA at pre op, 1 month post op and 12 months post op, according to grade of the ellipsoid zone defects.

Fig 4a. demonstrates the visual field of a 69 year old patient with mild L-ORD, showing well preserved peripheral vision, but who has a central scotoma (ellipsoid zone grade 3, BCVA $0.2 \log M A R)$. Fig 4b. demonstrates the visual field of a 70 year old patient with advanced L-ORD, who only has a temporal island of residual visual field remaining (ellipsoid zone grade 1, BCVA 1.5 LogMAR)

Fig 5a. Anterior lens capsule from a patient with L-ORD stained for wild type C1QTNF5. The capsule epithelium cytoplasm stains brown (arrowed) for the C1QTNF5 protein. Fig 5b demonstrates a negative control from the same patient. (original magnification $\times 20$ )

Fig 6 a) and b). Scanning electron micrographs of capsules from two different patients with L-ORD, demonstrating the helical structure of the free zonules. White arrow in Fig 6a denotes the capsule edge and black arrow denotes a zonular fibre. The arrows in fig $6 b$ denote examples of some of the individual fibrils making up a zonular fibre. Solid bar represents 10 microns.

Fig 7. Scanning electron micrograph of a capsule from a patient with L-ORD showing the area of zonular insertion. Solid bar represents 10 microns.

Fig 8. Scanning electron micrograph of a capsule from a patient with L-ORD showing the area of the zonule insertion and in particular, small fibrils from the under surface of the zonules blending with the underlying capsule. Solid bar represents 10 microns. 
Fig 9. Scanning electron micrograph of a capsule from a patient with L-ORD showing the area of the zonule insertion and the blending of the terminal zonule with the capsule. . Solid bar represents 5 microns 